\title{
La dynamique des systèmes de production dans les terroirs agricoles riverains de la forêt intercommunale de Fita-Agbado dans les communes de Dassa- Zoumé et de Savalou, Département des Collines au Centre du Bénin
}

\author{
Gaston S. AKOUEHOU ${ }^{1 *}$, Aimé HOUNDONOUGBO ${ }^{2}$ et Brice TENTE ${ }^{2}$ \\ ${ }^{1}$ Direction Générale des Forêts et des Ressources Naturelles, 02BP 1422, Bénin. \\ ${ }^{2}$ Université d'Abomey-Calavi, Faculté des Lettres, Arts et Sciences Humaines, \\ Département de Géographie et Aménagement du Territoire, Bénin. \\ *Auteur correspondant ; E-mail : akouehougas@yahoo.fr
}

\section{RESUME}

Les espaces forestiers du Département des Collines en général et des Communes de Dassa-Zoumè et de Savalou en particulier sont soumis à une pression agricole et une sur exploitation. Cette situation est la conséquence de la forte pression démographique et la dynamique des systèmes de production qui caractérisent cette région. La présente étude vise à étudier la dynamique socio-économique des systèmes de production agricole (SPA) des terroirs riverains de la forêt intercommunale de Fita des Communes de Dassa-Zoumè et de Savalou. Pour répondre à cette problématique, le milieu d'étude a été subdivisé en trois (03) zones suivant la taille des exploitations, la superficie emblavée et l'existence ou non de la jachère. Dans chaque zone, une auréole a été faite. L'approche systémique a permis de catégoriser les systèmes de production agricole par zone et d'analyser leur performance agro économique. Le traitement des données collectées a été fait avec le logiciel SPHINX PLUS ${ }^{2}$. Les résultats révèlent trois (3) systèmes de production agricole (SPA 1, SPA 2 et SPA 3). Les SPA 1 et 2 sont répétés dans les trois zones à l'exception du SPA 3 qui n'a été identifié que dans la zone 3. Les rendements des principales cultures varient d'un système de production agricole à un autre et la superficie cultivée est un facteur déterminant du revenu. Enfin, cette étude a montré que les systèmes de production sont caractérisés par une agriculture traditionnelle qui reste une source de revenu importante et de dégradation des espaces après l'exploitation forestière pour la fabrication du charbon de bois.

(C) 2013 International Formulae Group. All rights reserved.

Mots clés : Couvert forestier, système, production agricole.

\section{INTRODUCTION}

Après la tenue des sommets de Stockholm (Suède en 1972), de Rio de Janeiro (Brésil en 1992) et de Copenhague (Danemark en 2010), un accent particulier a été mis sur la gestion durable des ressources naturelles. De toutes ces ressources, celles forestières caractérisent les débats internationaux et nourrissent beaucoup de réflexions et d'actions (Glin, 2000). La foresterie et l'agriculture ont donc besoin d'être pleinement intégrées aux politiques de développement, en particulier les politiques agricoles (Lakany, 2004). En Afrique au sud du Sahara et au Bénin, le prélèvement des ressources forestières constitue l'activité principale des populations car le secteur primaire y est prédominant (Ezin, 2008). Mais face à l'explosion démographique, les ressources naturelles deviennent insuffisantes 
pour répondre aux besoins les plus essentiels des ménages agricoles des centres urbains et ruraux (FAO, 2000). Alors, l'homme a développé d'autres méthodes, techniques et stratégies pouvant lui permettre de tirer de son environnement les produits nécessaires à la satisfaction de ses besoins (Carol et Colfer, 2005). Malheureusement, ceci se fait de façon anarchique, entraînant la perte des ressources et la dégradation accélérée de l'environnement et surtout des terres agricoles. Bien que l'importance du rôle des forêts dans la lutte contre la pauvreté et la protection de l'environnement soit de plus en plus reconnue, la déforestation et la dégradation des forêts se poursuivent dans des proportions alarmantes (Lakany, 2004). Au Bénin, la superficie déboisée chaque année est estimée en moyenne à cent mille hectares (FAO, 2002). Au Bénin, la couverture forestière est passée de 4923000 ha en 1990 à 4625000 ha en 1995 soit une perte totale de 298000 ha de forêts en 5 ans (FAO, 2000). Le Bénin perd 65000 ha de forêt par an selon les estimations de la FAO (2011), soit un taux annuel de déforestation évalué à $1,3 \%$. Cette dégradation est d'autant plus inquiétante qu'elle ne laisse indifférents ni acteurs de développement, ni chercheurs (Boko et al., 2008).

Or, les pays africains sont caractérisés par une forte croissance démographique dont les effets sur l'environnement se traduisent par une dégradation accélérée des ressources naturelles (Gibigaye et al., 2007). La maîtrise de la déforestation exige le développement des systèmes agricoles qui n'entraînent pas seulement une amélioration des rendements agricoles, mais aussi une gestion durable des ressources naturelles. La présente étude a été réalisée dans la forêt intercommunale de Fita située dans le Département des Collines. Elle s'étend sur une superficie de 36885 ha dans les communes de Dassa-Zoumè et de Savalou. La zone d'étude se trouve entre les parallèles $7^{\circ} 34^{\prime}$ et $7^{\circ} 50^{\prime}$ de latitude nord et les méridiens $1^{\circ} 58^{\prime}$ et $2^{\circ} 08^{\prime}$ de longitude est (Figure 1). L'intérêt du choix de ce cadre repose sur le fait qu'il est une localité où les terres sont surexploitées et la pression s'exerce sur les ressources végétales. Il s'agit, d'une dynamique qui affecte dangereusement l'espace forestier avec toutes ses composantes et met en péril les générations futures. En effet l'objectif principal est d'étudier la dynamique socio-économique des systèmes de production agricole des différents terroirs de la forêt intercommunale de Fita. Il s'agit spécifiquement de: i) catégoriser les différents types de systèmes de production agricole des terroirs villageois riverains de la forêt intercommunale de Fita ii) étudier les caractéristiques agro-économiques des systèmes de production agricole et les impacts socio-économiques pour les exploitations agricoles.

\section{MATERIEL ET METHODES \\ Matériel de collecte de données}

Le matériel de collette de données est composé de: GPS (Global Positioning System) pour la prise des coordonnées des points stratégiques de la zone d'étude, machette et bottes, appareil photographique pour la prise des vues, fiches d'enquête et guide d'entretien élaborés à l'endroit des groupes cibles de la présente étude.

\section{Méthode \\ Echantillonnage}

Dans le cadre de ce travail il a été procédé dans un premier temps à un zonage du milieu d'étude. Le zonage a consisté à faire une auréole autour de la forêt en se basant sur la densité de la population, le nombre d'actifs agricoles, la formation végétale et l'existence ou non des jachères dans le système agraire. Ces critères ont été liés à d'autres critères comme la présence de réserves foncières, la riveraineté des villages. Ensuite, un sondage a été réalisé au niveau de chaque zone sur les ménages agricoles à partir du recensement de la population (INSAE, 2004). Sur un total de 1526 ménages agricoles recensés dans les villages, 195 ménages agricoles ont été enquêtés, soit un taux d'échantillonnage de $12,78 \%$. Le Tableau 1 présente la taille des échantillons par zone. 
Catégorisation des différents systèmes de production agricole des terroirs villageois riverains de la zone

Pour caractériser les systèmes de production agricole, les données suivantes ont été collectées : le genre, le statut de l'enquêté, la superficie emblavée, la taille de l'exploitation, les moyens de production, les associations et rotation de cultures, le mode d'accès à la terre et le choix du type de système de production. Ce qui a permis de faire ressortir les éléments caractéristiques de chacun des systèmes de production dans chaque zone et les facteurs qui affectent le développement ou non desdits systèmes.

Traitement et analyse des données socioéconomiques

Après les travaux de terrain, les données qui ont été recueillies ont subi une phase de restructuration. Il a fallu procéder d'abord au filtrage et à l'harmonisation des réponses afin de pouvoir les codifier. A l'aide du programme Excel 2007, un tableau de dépouillement a été réalisé. De ces tableaux, sont issus d'autres tableaux de synthèse. Enfin, avec le programme Excel 2007 des graphiques ont été réalisés à partir de ces tableaux pour servir d'illustration. Ces graphiques sont soumis à une analyse qualitative suivie de commentaire et d'interprétation. Les données qualitatives (données non mesurables) ont fait l'objet d'analyses statistiques de tableaux croisés à l'aide du SPHINX version 4.5. Il s'agit du croisement entre: actif agricole et les systèmes de production agricole, activités principales et les systèmes de production agricole, main-d'œuvre et zones, maind'œuvre et mode d'accès à la terre, maind'œuvre et statut, rendement des cultures et les systèmes de production agricole, les revenus annuels des exploitants agricoles et zones, les revenus annuels des exploitants forestiers et type de zonage. Le calcul des moyennes et des erreurs-types a été fait avec le logiciel SPHINX.

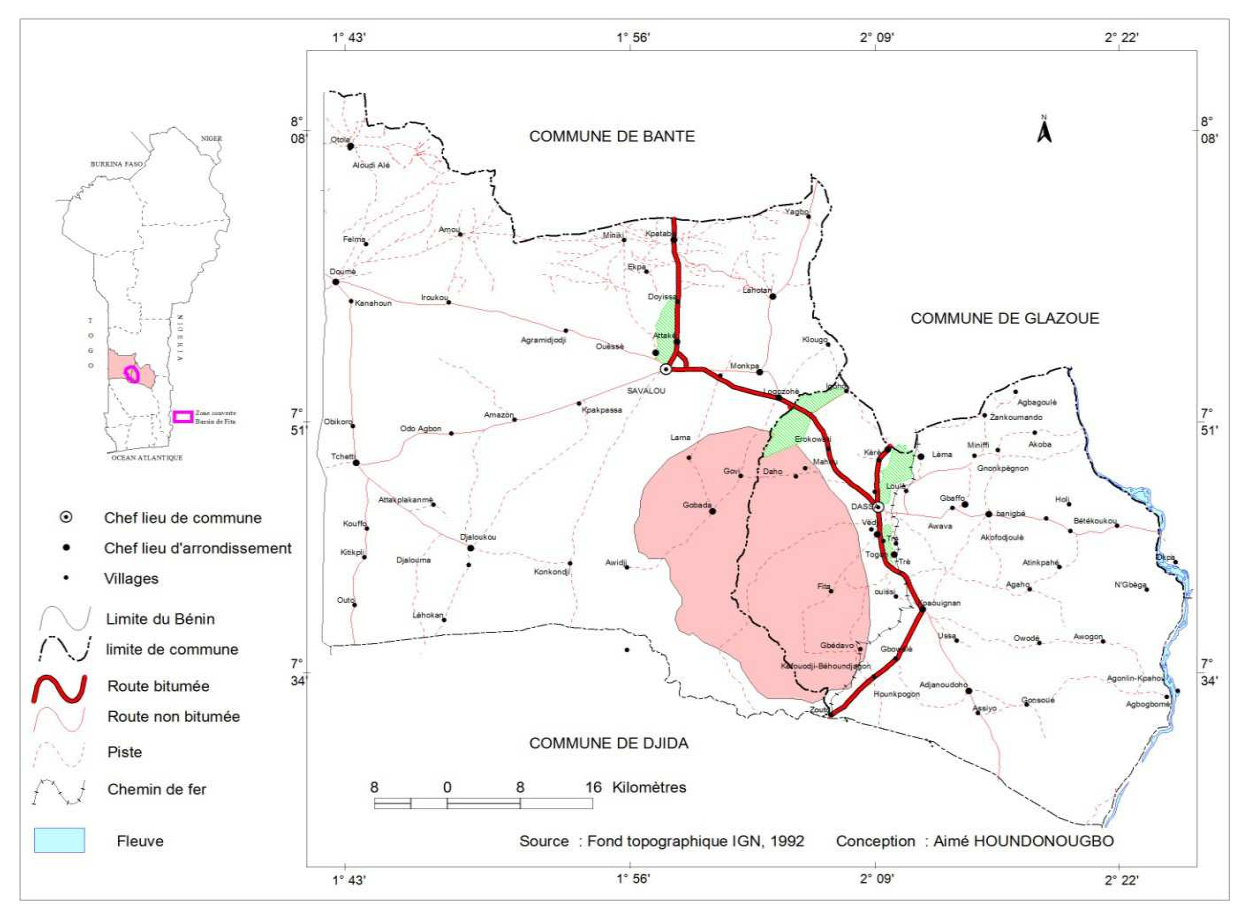

Figure 1 : Situation géographique de la forêt intercommunale de Fita-Agbado au Bénin. 


\section{RESULTATS}

Les résultats obtenus s'articulent autour de deux (2) points: l'analyse des systèmes de production agricole et leurs performances agro-économiques.

\section{Catégorisation des systèmes de production} agricole

Les types de systèmes de production identifiés

Trois types de systèmes de production sont identifiés dans à Fita subdivisée en trois zones agro-socio-écologique (Figure 2). Il présente les types de système de production agricole et leurs caractéristiques.

\section{Système de production agricole de type $\mathbf{n}^{\circ} 1$} (SPA 1)

Il est pratiqué par $64,39 \%$ de la population échantillonnée dans les trois zones d'étude. Ce type de système de production est composé des exploitations agricoles disposant d'une superficie comprise entre 0 et 5 ha avec une taille comprise entre 2 et 5 actifs agricoles. La superficie emblavée chaque année dans ces exploitations est inférieure à 5 ha. L'activité principale des actifs agricoles dans ce cas est l'agriculture tandis que la carbonisation est l'activité secondaire. Le mode d'accès à la terre est le don et l'héritage. Les moyens de production identifiés ont été essentiellement manuels. C'est donc, un système de production agricole caractérisé par une agriculture traditionnelle.

\section{Système de production agricole de type $n^{\circ} 2$ (SPA 2)}

Il est pratiqué par $28,78 \%$ des exploitations agricoles enquêtées. Ce type de système de production est composé des exploitants agricoles disposant d'une superficie comprise entre 5 et 10 ha et est pratiqué dans les trois zones d'étude. Le mode d'accès à la terre dans ce type de système de production est : l'achat, l'héritage et le don avec une taille d'actifs agricoles comprise entre 5 et 10. La superficie moyenne emblavée chaque année est de 5 ha. L'activité principale est l'agriculture et l'activité secondaire est la carbonisation. Le SPA 2 est caractérisé par la polyculture sans légumineuses.

\section{Système de production agricole de type $\mathrm{n}^{\circ} 3$ (SPA 3)}

Le SPA 3 est pratiqué par les 6,83\% de la population échantillonnée. Ce type de système de production est composé des exploitants disposant d'une superficie supérieure à 10 ha rencontré uniquement dans la zone 3 . Le mode d'accès à la terre est l'achat et l'héritage avec une taille d'actifs agricoles comprise entre 5 et 10. La moyenne de la superficie emblavée chaque année est de 7 ha. Leurs activités principales sont l'agriculture et la carbonisation avec des espèces de valeur: Ptérocarpus erinaceus, Anogeissus leiocarpus, Prosopis africana, Combretum sp. Pseudocedrela kotchyi, Diospiros mespiliformis, Burkea africana, Isoberlinia doka, Afzelia africana.

Dans ce type de système de production agricole dans la zone 3, la présence des cours d'eau tels que : Zou, Amonsouhanou, Toto et Idjesso fait que les chefs d'exploitations agricoles qui sont des allochtones venant du Département du ZOU (communes de Zakpota et Djidja) achètent la terre auprès du Roi de Fita.

Modes d'accès à la terre dans chaque Système de production agricole (SPA)

Dans la zone d'étude, les modes d'accès à la terre varient d'un système de production agricole à un autre. Il s'agit principalement de l'héritage, du don et de l'achat. La Figure 3, présente la répartition des exploitations agricoles enquêtées en fonction des modes d'accès à la terre. L'héritage est le mode d'accès à la terre le plus répandu car il est pratiqué par au moins $60 \%$ de la population échantillonnée. Le don est aussi présent dans les trois systèmes de production agricole. Les exploitants agricoles qui en jouissent sont moins importants et varient entre $3 \%$ et $25 \%$. Par ailleurs, $18 \%$ des exploitants achètent les terres agricoles au 
près du Roi de Fita. L'achat est le mode d'accès le moins adopté et ne concerne que les systèmes de production agricole 1 et 2 . A Fita et dans les villages ou hameaux riverains, l'accès à la terre par le don et l'achat s'effectue avec l'accord du chef traditionnel (le roi de Fita, chef de terre). Le demandeur ou l'acquéreur devra conformément à la tradition offrir des boissons, des animaux domestiques tels que les moutons et coqs parfois une somme d'argent variant entre 15000 FCFA et 50000 CFA pour recevoir les bénédictions et la protection des ancêtres. A Gobada, l'accès à la terre présente des caractéristiques similaires à celles identifiées à Fita, à la seule différence que, la propriété foncière est exercée par le chef de collectivité et non un roi. Cependant, à Kéfouodji, l'autorité administrative exercée par les élus locaux ou chefs de villages est contrebalancée par un pouvoir foncier très étendu et détenu par les chefs de hameaux ou "Gohonon". Ainsi, les terres forestières ou ressources naturelles sont gérées de façon endogène. L'agriculture dans cette localité utilise des techniques peu recommandées : les techniques itinérantes sur brûlis. Tout ceci contribue à la dégradation des ressources naturelles.

Caractéristiques agro-économiques des systèmes de production agricole Actifs agricoles au niveau des SPA

Les actifs agricoles varient en fonction des systèmes de production agricole dans le bassin de production de Fita-Agbado. Le Tableau 3 indique leur répartition. L'analyse des données montre que:

- Au niveau des SPA 1, dans les villages de Govi, Zadowin, Daho et Gbédavo, plus de $77 \%$ des exploitants agricoles enquêtés ont un nombre d'actifs agricoles inférieur à 3 et la superficie emblavée chaque année est de 3 ha.

- Au niveau des SPA 2, dans les villages de Fita Daho, Gobada et Zouto, plus de 57\% des exploitants agricoles ont un nombre d'actifs agricoles compris entre [2,5[; la superficie emblavée par an est au plus de 5 ha.
- Au niveau des SPA 3, dans les villages de Fita, et Kéfouodji, les $100 \%$ des exploitants agricoles ont un nombre d'actifs agricoles supérieur ou égal à 10 . La superficie emblavée par an est de 7 ha. Au total, les actifs agricoles sont moins nombreux pour le SPA 1 et plus nombreux dans le SPA 3 de la zone agrosocio-écologique 3.

\section{Activités principales au niveau des SPA}

Les activités principales varient en fonction des systèmes de production agricole dans le bassin de production agricole de FitaAgbado. Il s'agit de l'agriculture, de l'exploitation forestière, de Taxi-moto, de la carbonisation et autres activités secondaires. La Figure 3 présente la répartition des exploitations en fonction des activités principales au niveau de chaque SPA. La superficie emblavée chaque année est plus importante dans la zone 3 que les autres zones. En effet, lorsque le nombre d'actifs agricoles est plus important, la superficie emblavée l'est aussi. La Figure 4 présente les activités principales dans chaque système de production. L'agriculture est l'activité principale pratiquée au niveau des trois SPA. Au niveau des SPA 2 de la zone 2, toutes les activités identifiées sont pratiquées. Par contre, aucun des Chefs d'exploitations des SPA 1 et 2 ne pratique le taxi-moto. Cependant, les deux activités pratiquées au niveau des SPA 3 sont: la carbonisation et l'agriculture pratiquées par $50 \%$ les exploitations. Ces exploitations ont emblavé en 2009, 17702 ha représentant $48 \%$ des terres. La superficie restante dans la zone d'étude est occupée par les forêts qui sont suffisamment anthropiques et les zones d'habitations. Le SPA 3 qui se rencontre dans la zone 3 mérite un suivi à cause de la carbonisation qui prend plus d'ampleur.

\section{Force de travail}

L'étendue et la rentabilité des terres cultivées dépendent du type de main-d'œuvre utilisé et varient d'une zone à une autre. La main-d'œuvre varie d'une zone à une autre.

Le Tableau 4 présente la main-d'œuvre utilisée par zone. L'utilisation de la main- 
d'œuvre salariale est la plus importante dans les trois zones, mais elle est plus utilisée dans la zone $3(86 \%)$. La main-d'œuvre familiale est aussi utilisée dans les trois zones. Mais ce type de main-d'œuvre est plus utilisé dans la zone $1(33,3 \%)$.

Le Tableau 5 présente la répartition de la main-d'œuvre en fonction du mode d'accès à la terre. De l'analyse des données de ce tableau, il ressort que la main-d'œuvre familiale est adoptée par les exploitations agricoles sauf dans le cas de l'achat où tous les exploitants utilisent uniquement la maind'œuvre occasionnelle (100\%). Par contre, les exploitants qui ont acquis leurs terres par le don utilisent plus la main-d'œuvre familiale $(60 \%)$. Le Tableau 6 traduit la variation de la main-d'œuvre en fonction des statuts des exploitants. Mais, suivant que l'exploitant est migrant ou autochtone, il y a une différence dans l'adoption de la main-d'œuvre. 50\% des migrants optent pour l'utilisation de la maind'œuvre occasionnelle et de la main-d'œuvre familiale.

\section{Rendements des principales cultures pratiquées}

Les principales cultures pratiquées dans la zone étudiée sont: le maïs, l'igname, l'arachide, le soja, le sorgho et le niébé. Les rendements de ces différentes cultures à l'hectare dans les SPA sont représentés par les Figures 5, 6, 7, 8 et 9.

\section{Cas du maïs}

La Figure 5 traduit les rendements du maïs (kg/ha) par système de production. De l'analyse des données, il ressort que le rendement du maïs le plus élevé est obtenu au niveau du SPA 3 dans la zone 3 car $50 \%$ des producteurs enquêtés affirment que dans ce type de SPA, ils obtiennent un rendement supérieur à $1000 \mathrm{~kg} / \mathrm{ha}$. Au niveau du SPA 2 dans la zone $1,38 \%$ des producteurs estiment avoir un rendement inférieur à $300 \mathrm{~kg} / \mathrm{ha}$ contre seulement $8 \%$ au niveau des SPA 1 de la zone 2. La moyenne des erreurs commises sur chaque producteur de maïs est égale à $5 \%$. Les observations participantes ont montré par contre que les techniques culturales et les affleurements rocheux, favorisant l'installation des sols minéraux bruts inaptes à l'agriculture, sont à la base du rendement médiocre obtenu dans la zone 2.

\section{Cas du niébé}

La Figure 6 présente les rendements du niébé (kg/ha) par système de production agricole. De l'analyse des données, il ressort que près de $75 \%$ des exploitations des SPA 3 de la zone 3 n'ont aucune idée du rendement du niébé en $\mathrm{kg} / \mathrm{ha}$. Le rendement le plus bas est obtenu dans les SPA 2 de la zone $1: 30 \%$ des exploitations ont une production de moins de $300 \mathrm{~kg} / \mathrm{ha}$. Mais les exploitants du SPA 1 de la zone 2 enregistrent les meilleurs rendements : 9\% des producteurs affirment avoir obtenu plus de $1000 \mathrm{~kg} / \mathrm{ha}$. La moyenne des erreurs commises est de $0,26 \%$.

\section{Cas du sorgho}

La Figure 7 traduit les rendements du sorgho $(\mathrm{kg} / \mathrm{ha})$ par système de production agricole. Le sorgho est très peu produit dans la zone de l'étude. Le rendement le plus important est obtenu au niveau des SPA 2 de la zone 2: $46 \%$ des exploitations ont un rendement moyen de $400 \mathrm{~kg} / \mathrm{ha}$. Les $25 \%$ des exploitations au niveau du SPA 3 de la zone 3 ont un rendement supérieur à $1000 \mathrm{~kg} / \mathrm{ha}$, mais seulement $18 \%$ des exploitations des SPA 1 de la zone 1 ont un rendement moyen de $850 \mathrm{~kg} / \mathrm{ha}$. La moyenne des erreurs commises sur chaque producteur de sorgho est égale à : 0,53\%. Par ailleurs, au niveau des trois SPA plus de $45 \%$ des exploitations n'ont pas pu estimer le rendement du sorgho en $\mathrm{kg} / \mathrm{ha}$.

\section{Cas du soja}

La Figure 8 traduit les rendements du soja $(\mathrm{kg} / \mathrm{ha})$ par système de production agricole. L'analyse des données a montré que le rendement du soja le plus important $(>1000$ $\mathrm{kg} / \mathrm{ha}$ ) est observé au niveau du SPA 1 de la zone 1 avec $25 \%$ des exploitants. Le SPA 2 de la zone 2 vient en deuxième position en termes de rendement (22\% des exploitants ont enregistré un rendement supérieur à 1000 $\mathrm{kg} / \mathrm{ha}$ ). Au niveau des SPA 3 dans la zone 3, 
le rendement le plus important de cette culture est une moyenne de $850 \mathrm{~kg} / \mathrm{ha}(27 \%$ des exploitants). Par contre, $50 \%$ des exploitants des SPA 3 de la zone 3 n'ont aucune idée du rendement de cette culture en $\mathrm{kg} / \mathrm{ha}$. Le faible taux de production du soja dans la zone 3 serait à la base des non réponses observées. $\mathrm{La}$ moyenne des erreurs commises est égale à $0,25 \%$.

\section{Cas de l'arachide}

La Figure 9 traduit les rendements de l'arachide $(\mathrm{kg} / \mathrm{ha})$ par système de production agricole. L'arachide est produite au niveau de tous les systèmes de production agricole avec un rendement supérieur à $1000 \mathrm{~kg} / \mathrm{ha}$ obtenu par au moins $25 \%$ des exploitants. La moyenne des erreurs commises est de $1,26 \%$. Tous les systèmes de production agricole ont donc les meilleurs rendements en ce qui concerne cette culture.

\section{Revenus issus de l'agriculture}

La Figure 10 représente les revenus des exploitations agricoles enquêtées en fonction des zones. L'analyse des données à montré que les revenus agricoles annuels des exploitants varient d'une zone à une autre. Ils sont compris entre 100000 et 400000 F CFA par an, mais plus importants dans la zone 3 que dans les deux autres zones. En effet, $42 \%$ des exploitations enquêtées ont des revenus annuels moyens estimés à $300000 \mathrm{~F}$ CFA dans la zone 3. Par contre, $36 \%$ des exploitants de la zone 2 ont un revenu supérieur à $400000 \mathrm{~F} \mathrm{CFA}$ et seulement, 25\% des exploitations de la zone 1 ont un revenu annuel de 300000 F CFA. Ainsi, les revenus issus de l'agriculture ne permettent pas aux exploitants agricoles d'épargner ou d'améliorer leur niveau de vie (résultat reconnu par les $50 \%$ de l'échantillon). L'agriculture permet au ménage, de disposer de produits vivriers qui ne suffisent pas parfois pour couvrir la période de soudure. Par conséquent, certaines exploitations abandonnent l'agriculture au profit d'autres activités telles que le taxi-moto, à Fita, le concassage de pierres dans le village de Daho et la carbonisation dans les villages de Zouto et de Gbédavo. Ce qui confirme l'hypothèse selon laquelle l'efficacité agroéconomique du système de production dépend des systèmes de culture pratiqués par les populations et la proximité de la zone boisée. Les essences utilisées pour la carbonisation sont Ptérocarpus erinaceus, Anogeissus leiocarpus, Prosopis africana, Combretum sp. Pseudocedrela kotchyi, Diospiros mespiliformis, Burkea africana, Isoberlinia doka Afzelia africana... Les espèces en voie de disparition signalées sont Pterocarpus erinaceus et Prosopis africana. Les techniques de carbonisation utilisées sont les meules traditionnelles disséminées dans les champs et les jachères. Avec ces techniques, les spécialistes (qui à Gobada sont surtout des allochtones de Zakpota et de Djidja) fabriquent de charbon toute l'année mais surtout pendant la saison sèche (Photo du marché rural de Adjakonou). L'utilisation de la tronçonneuse par les jeunes du milieu favorise cette activité malgré la répression des agents forestiers.

\section{Etude comparative des revenus issus de l'agriculture et de la carbonisation}

La Figure 11 présente l'étude comparative des revenus issus de l'agriculture et de la carbonisation. De l'analyse des données, il est ressorti que dans la zone 1 le revenu issu de l'agriculture est $300000 \mathrm{~F}$ CFA tandis que celui issu de la carbonisation est de 500000 F CFA par an. Dans la zone 2, le revenu issu de l'agriculture est de 400000 F CFA par contre celui issu de la carbonisation est de 500000 F CFA. Quant à la zone 3 le revenu issu de l'agriculture est de $150000 \mathrm{~F}$ CFA par contre le revenu issu de la carbonisation est de 300000 F CFA. Dans toutes les zones, les revenus issus de la fabrication du charbon sont plus importants que les revenus issus de l'agriculture. 


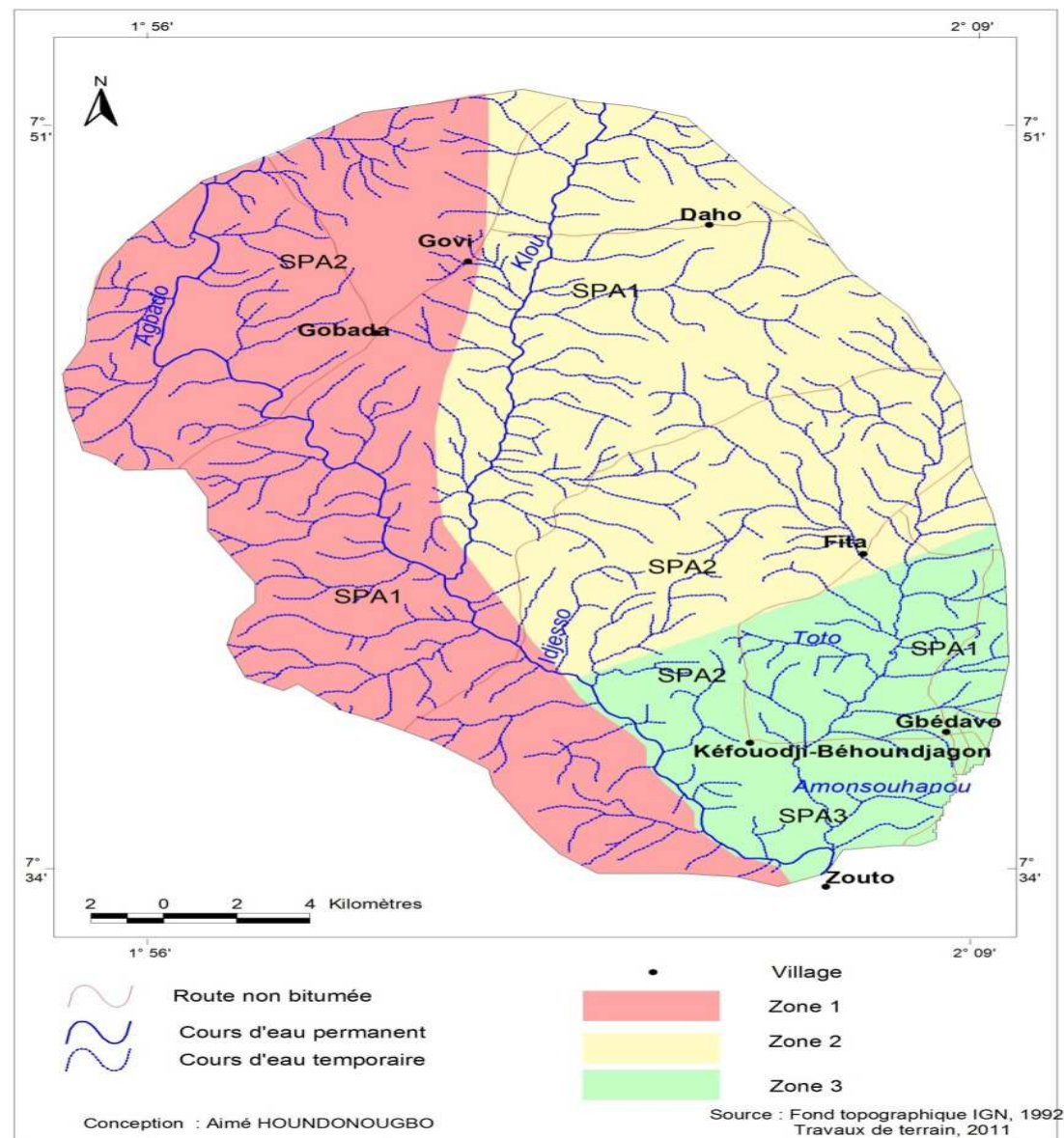

Figure 2 : Zonage et hydrographie de la forêt intercommunale de Fita.

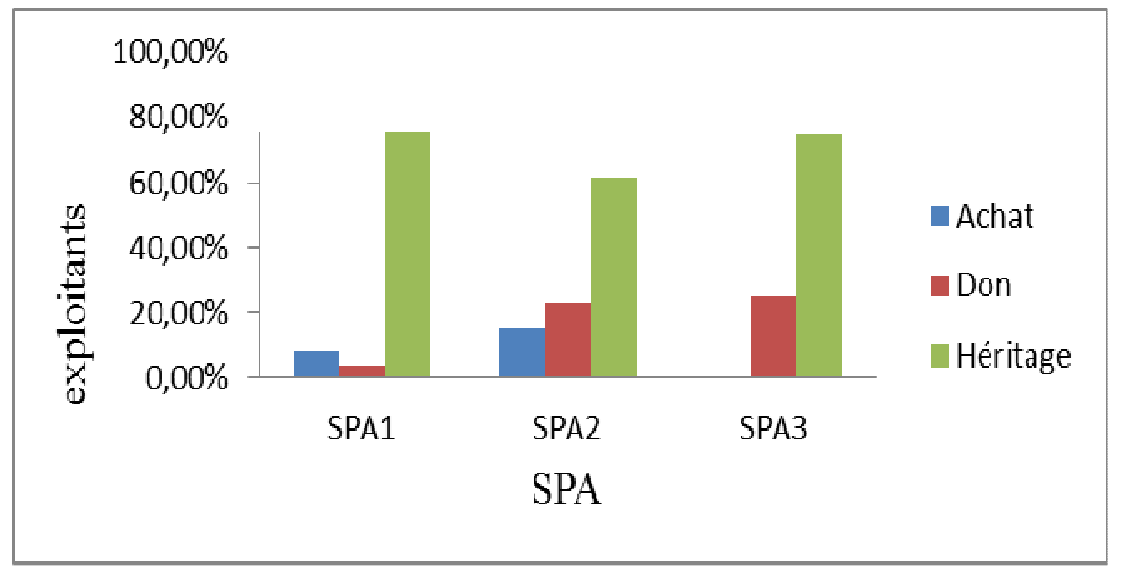

Figure 3: Répartition des exploitants en fonction des modes d'accès à la terre agricole dans chaque SPA. 


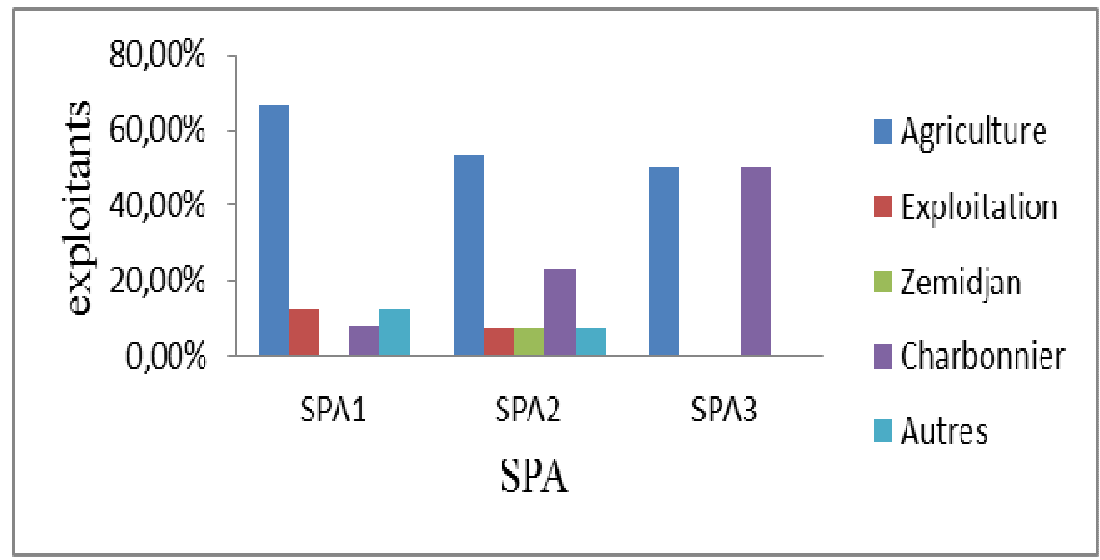

Figure 4 : Activités principales au niveau de chaque SPA.

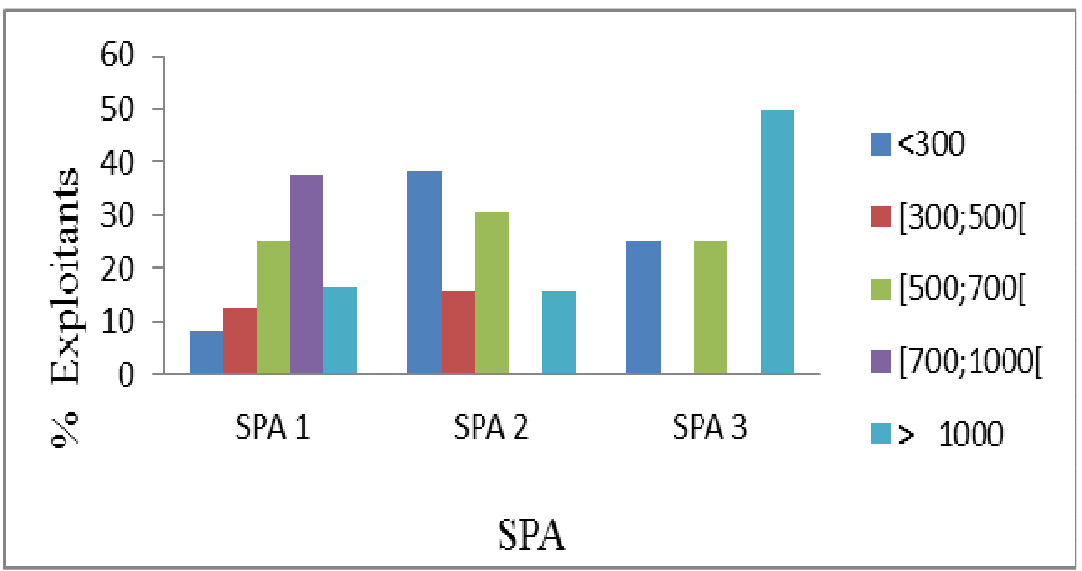

Figure 5: Rendement du maïs (kg/ha) par système de production.

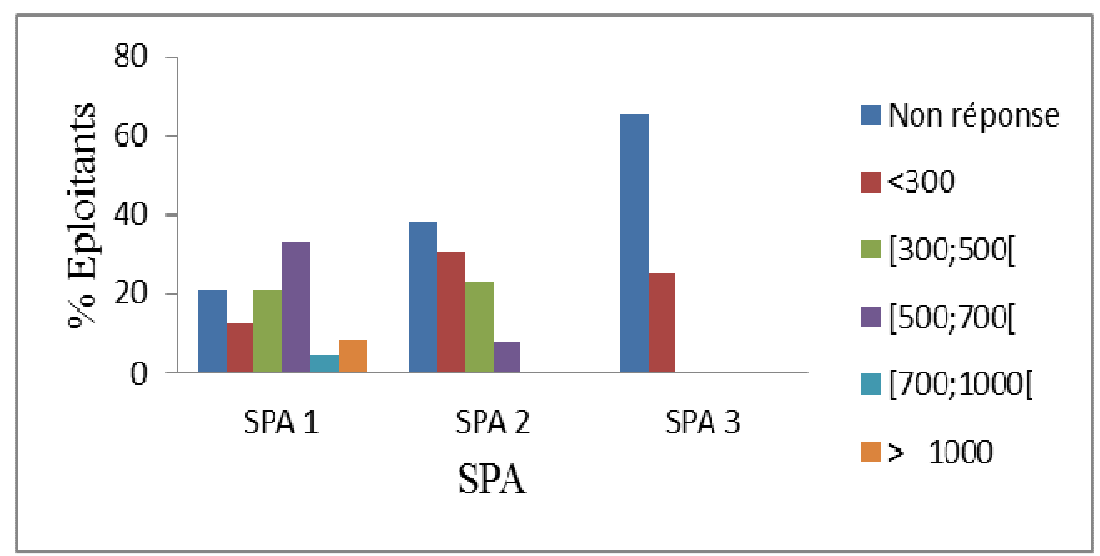

Figure 6: Rendements du niébé (kg/ha) par système de production. 


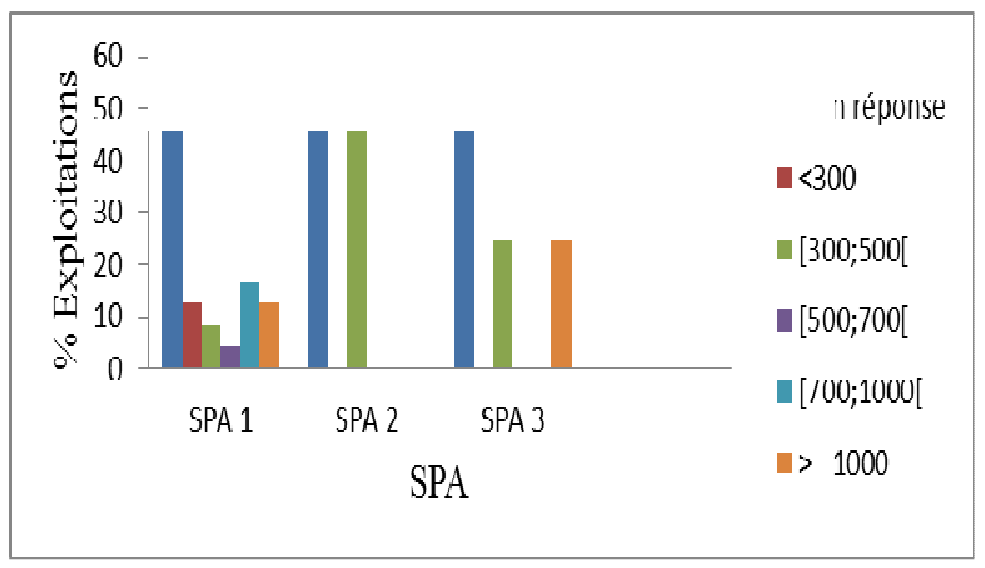

Figure 7: Rendements du sorgho $(\mathrm{kg} / \mathrm{ha})$ par système de production.

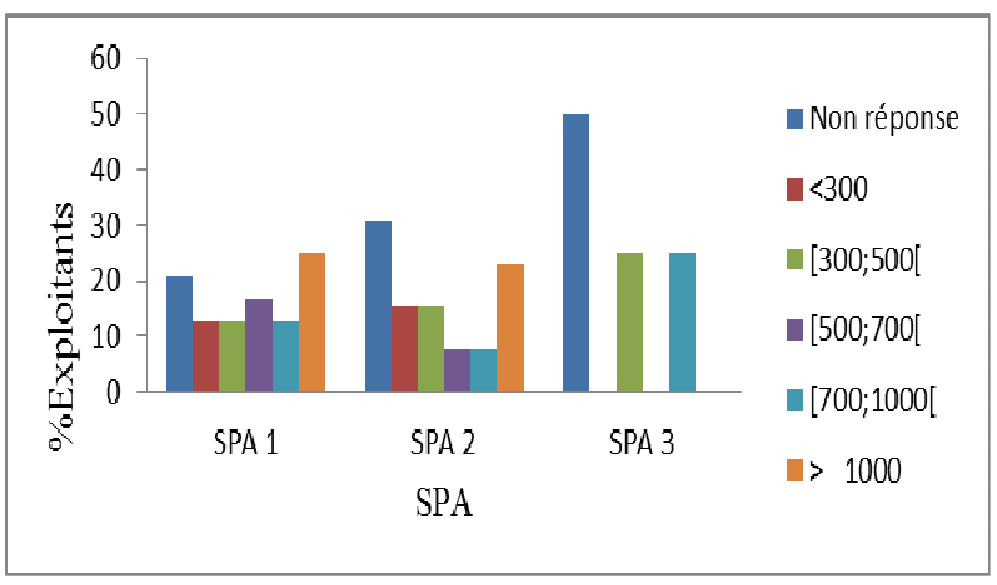

Figure 8: Rendements du soja (kg/ha) par système de production.

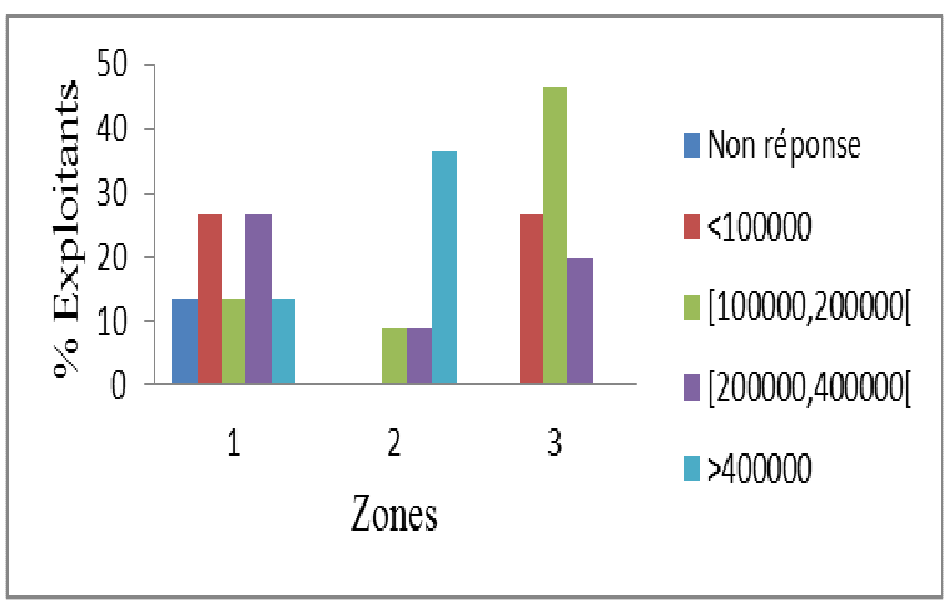

Figure 9: Rendements de l'arachide par SPA. 


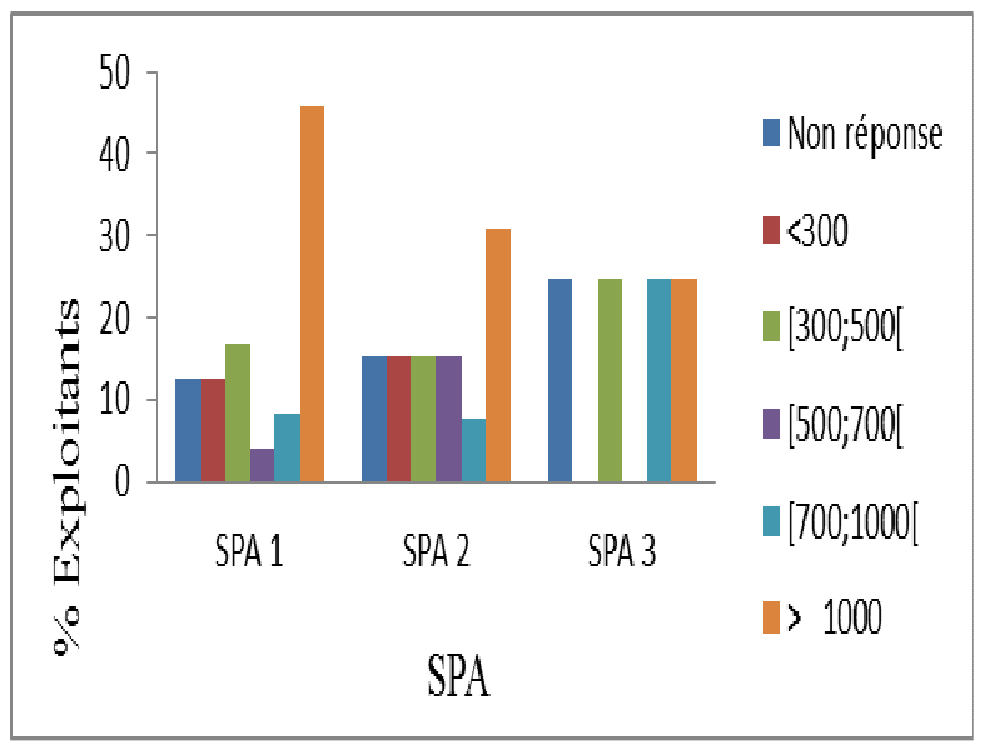

Figure 10: Revenus issus de l'agriculture par zone.

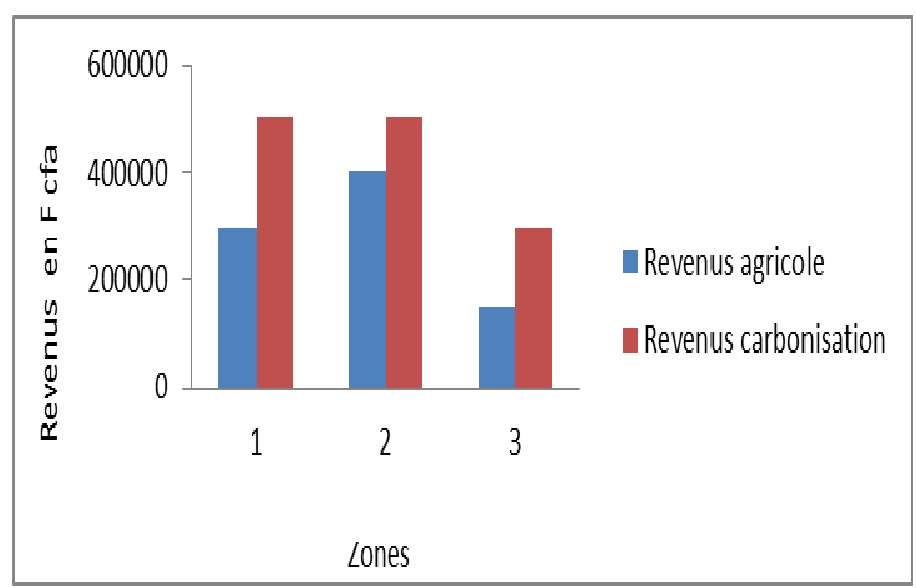

Figure 11: Etude comparative des revenus issus de l'agriculture et de la carbonisation.

Tableau 1 : Taille des échantillons en fonction des zones.

\begin{tabular}{lccc}
\hline Zones & $\begin{array}{c}\text { Nombre total de } \\
\text { ménages recensés }\end{array}$ & $\begin{array}{c}\text { Nombre de ménages } \\
\text { enquêtés }\end{array}$ & $\begin{array}{c}\text { Taux d'échantionnage } \\
(\%)\end{array}$ \\
\hline 1 & 557 & 59 & 10,6 \\
2 & 469 & 63 & 13,43 \\
3 & 500 & 73 & 14,6 \\
Total & 1526 & 195 & 12,78 \\
\hline
\end{tabular}


Tableau 2: Catégorisation des systèmes de production agricole à Fita.

\begin{tabular}{cccc}
\hline Zon & $\begin{array}{c}\text { Systèmes } \\
\text { production }\end{array}$ & $\begin{array}{c}\text { Superficies cultivées } \\
\text { en ha }\end{array}$ & Modes d'accès à la terre \\
\hline \multirow{2}{*}{1} & SPA1 & 0 à 5 & Héritage \\
& SPA2 & 5 à 10 & Achat, don, héritage \\
\hline \multirow{2}{*}{3} & SPA1 & 0 à 5 & Don, héritage \\
3 & SPA2 & 5 à 10 & Achat, don, héritage \\
\hline & SPA1 & 0 à 5 & Don, héritage \\
& SPA2 & 5 à 10 & Don, héritage \\
Achat, héritage
\end{tabular}

$\mathrm{SPA}=$ Système de Production Agricole

Tableau 3 : Proportions d'actifs agricoles par SPA.

\begin{tabular}{lccc}
\hline Catégories d'actifs agricoles & SPA 1/ \% & SPA2/ \% & SPA3/ \% \\
\hline$<3$ & 77,3 & 18,2 & 4,5 \\
{$[2,5[$} & 35,7 & 57,1 & 7,1 \\
{$[5,10[$} & 50 & 25 & 25 \\
$>10$ & 0 & 0 & 100 \\
\hline
\end{tabular}

Tableau 4: Répartition de la main-d'œuvre par zone.

\begin{tabular}{lcc}
\hline \multirow{2}{*}{ Zones } & \multicolumn{2}{c}{ Type de main-d'œuvre } \\
\cline { 2 - 3 } & Occasionnelle (\%) & Familiale (\%) \\
\hline 1 & 66,7 & 33,3 \\
2 & 72,7 & 27,3 \\
3 & 86 & 14 \\
\hline
\end{tabular}

Tableau 5: Type de main-d'œuvre en fonction du mode d'accès à la terre.

\begin{tabular}{lcc}
\hline Modes d'accès à la terre & \multicolumn{2}{c}{ Type de main-d'ouvre } \\
\cline { 2 - 3 } & Occasionnelle (\%) & Familiale (\%) \\
\hline Achat & 100 & 0 \\
Don & 40 & 60 \\
Héritage & 78,1 & 21,9 \\
\hline
\end{tabular}


Tableau 6: Variation de la main-d'œuvre en fonction du statut de l'exploitant.

\begin{tabular}{lcc}
\hline Statut & \multicolumn{2}{c}{ Type de main-d'œuvre } \\
\cline { 2 - 3 } & Occasionnelle (\%) & Familiale (\%) \\
\hline Autochtone & 21,6 & 78,4 \\
Migrant & 50 & 50 \\
\hline
\end{tabular}

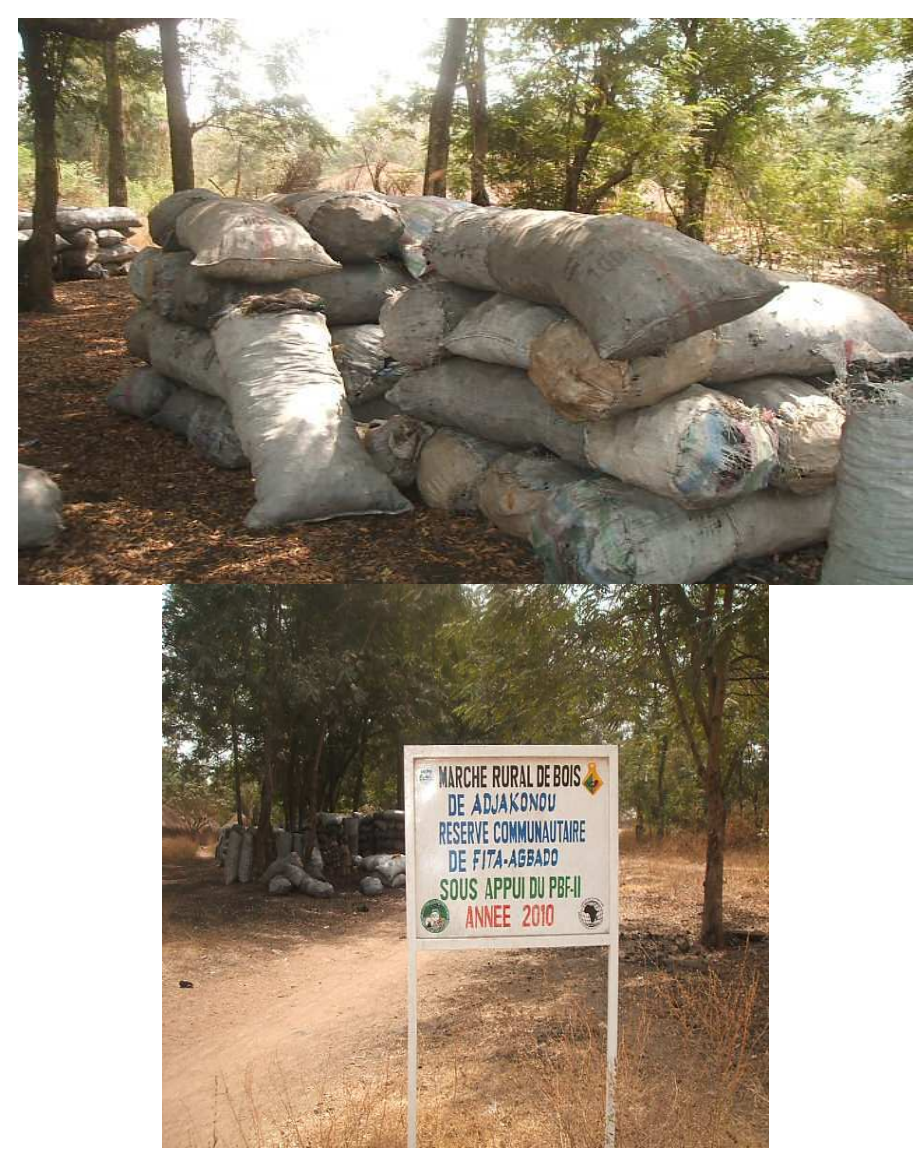

Photo : Charbon de bois sur le site de vente d'Adjakonou.

\section{DISCUSSION}

Il ressort des résultats que les systèmes de production agricole des terroirs riverains de la forêt intercommunale de Fita-Agbado dans les communes de Dassa et de Savalou sont semblables aux systèmes traditionnels améliorés tels que définis par Avononmadegbé (2011). L'agriculture et la carbonisation sont les principales activités des populations actives exploitantes des SPA de la zone d'étude. L'agriculture et la carbonisation sont pratiquées par $50 \%$ des enquêtés. Assouni (2009), dans une étude sur les impacts socio-économiques et environnementaux de l'exploitation du bois dans la commune de Tchaourou, en arrive à la même conclusion. En effet, il a indiqué que la culture itinérante sur brûlis y est répandue avec l'utilisation des outils rudimentaires comme la houe, le coupe-coupe, la hache, etc. 
Ainsi, les résultats des enquêtes quantitatives et les entretiens semi-structurés ont également montré que lorsque les systèmes d'exploitation sont limités à un facteur de production (terre, moyen de production), les exploitants développent des logiques endogènes. Ce qui confirme les analyses de Akouèhou (2004) dans la région des Monts Kouffé au Bénin.

L'agriculture telle que menée actuellement dans les terroirs riverains de la forêt intercommunale de Fita Agbado porte préjudice à sa performance agro-économique. La main-d'œuvre utilisée varie d'un système de production agricole à un autre et est occasionnelle au moins à $66 \%$ dans tous les SPA. En plus, les exploitations agricoles des zones étudiées intègrent peu de pesticides et d'engrais chimiques malgré le faible rendement observé. Mais, les principales cultures pratiquées par les exploitants agricoles enquêtés sont : le maïs, le niébé, le soja, le sorgho, et l'arachide (avec un rendement qui varie d'une zone à l'autre). Les cultures les plus rentables (maïs et arachide) ont des rendements parfois supérieurs à 1000 $\mathrm{kg} /$ ha pour le maïs selon $50 \%$ des enquêtés et pour l'arachide selon au moins $25 \%$ des enquêtés. Les raisons qui justifient cette baisse de rendement seraient, la qualité des sols et les techniques agricoles utilisées. Heldmann et Doevenspeck (2009) dans son étude comparative explique que les conditions biogéographiques, l'insolation, la température et l'humidité relative au Bénin sont des facteurs physiques favorables à la croissance végétative.

\section{Conclusion}

L'agriculture dans ce contexte de subsistance doit considérer la gestion des écosystèmes forestiers et la conservation de la diversité biologique. Il s'agira de repenser un autre mode de gestion de la forêt intercommunale de Fita-Agbado afin de l'intégrer au circuit de développement des communes de Dassa-Zoumè et de Savalou. Cette nouvelle conception de gestion impose aux communes concernées un mode particulier de planification spatiale et de gestion durable des ressources naturelles. Mais, la dynamique de l'utilisation des terres a montré que la zone d'étude connait une réduction des formations forestières fermées telles que les forêts galeries et les forêts claires au profit des savanes arbustives qui deviennent dominantes. La présence des zones de cultures montre l'importance la dégradation des ressources naturelles comme signalé dans les mêmes conditions par Akouèhou et al. (2012) autour de la forêt communautaire de Tfoungou dans la commune de Djougou Département de la Donga (Benin). En effet dans les terroirs riverains de cette forêt communautaire de Tfoungou, les moyens de production sont manuels et rudimentaires (coupe-coupe, houe, daba, etc.) et la taille des exploitations varie de 2 à 5 ha. Mais, le facteur incitateur à la destruction des forêts est la considération qu'en font les populations riveraines. Outre l'exploitation forestière anarchique dont font l'objet les ressources naturelles dans cette zone, les agriculteurs considèrent l'espace forestier comme une disponibilité en terres cultivables, réputé pour sa fertilité. Pour ces derniers, la forêt est une richesse naturelle à exploiter et intarissable. Ces constats confirment les observations faites par le même auteur dans la région des Monts Kouffé au Bénin (Akouèhou, 2004).

\section{REFERENCES}

Akouèhou SG, Gbozo E, Tente B, Houndonougbo A, Ganglo JC. 2012. Les systèmes de production agricoles et la gestion de la forêt communautaire de Tfoungou dans la commune de Djougou Département de la Donga (Benin). BenGéo. Revue Semestrielle de Géographie du Bénin. Université d'Abomey-Calavi, Bénin, 11: 21-39.

Akouèhou SG. 2004. Environnement institutionnel, et la gestion traditionnelle des espaces forestiers : cas de la région des Monts Kouffé au Bénin. Les Cahiers 
d'Outre-Mer. Revu de Géographie de Bordeaux, 155-173. Online.

Assouni J. 2009. Impacts socio-économiques et environnementaux de l'exploitation du bois dans la commune de Tchaourou, Mémoire de DEA, FLASH/UAC, 97p.

Avononmadegbe B. 2011. Interview accordée à Inter Press Service (IPS) dans le cadre du PGFTR (Programme de Gestion des Forêts et Terroirs Riverains), Cotonou, $2 \mathrm{p}$.

Boko M, Ogouwale E. 2008 : Environnement naturel et développement durable en Afrique. Support de cours, $24 \mathrm{p}$.

Colfer CJP. 2005. The Complex Forest: Communities, Uncertainty, and Adaptive Collaborative Management. Resources for the Future/CIFOR: Washington, DC.

Cenatel. 2002. Base de données géoréférencées sur l'utilisation agricole des terres au Bénin. Rapport final d'Etudes. DFRN/MAEP, Cotonou, 20p.

Ezin W. 2008. Pression démographique et dégradation des ressources naturelles dans la commune de Dassa-Zoumé : Cas de l'arrondissement de Paouignan, mémoire de maîtrise de géographie, option aménagement du territoire, UAC/FLASH, 112p.

FAO. 2000. Importance des forêts dans la lutte contre la pauvreté et protection de l'environnement. FAO, http:/ www. média-office @ fao.org.

FAO. 2011. Facteurs de dégradation de l'environnement à l'échelle planétaire; Rapport annuel, FAO, 210 p.

FAO. 2002. Evaluation des ressources forestières mondiale 2000. Rapport principal $\mathrm{N}^{\circ} 140$ de l'ONU pour l'alimentation et l'agriculture. Etude forêt, Rome 2002, 466p.
Gibigaye M, Sinsin B, Adegbidi. 2007. Activités économiques et ressources naturelles dans les forêts classées de Ouénou-Bénou. In Climat et Développement. LECREDE: Benin ; 68$76 \mathrm{pp}$.

Giorgi F, Mearns LO. 2002. Calculation of average, uncertainty range, and reliability of regional climate change from AOGCM simulations via the Reliability Ensemble Averaging (REA) method. J. Clim., 15: 1141-1158.

GLIN LC. 2000. Pour une gestion participative durable des resources naturelles au Bénin: étude de la viabilité des groupements forestiers de la forêt classée de Tchaorou-Toui-Kilibo. Thèse d'ingénieur agronome, FSA / UNB, Abomey-Calavi, Bénin.

Heldmann M, Doevenspeck M. 2009. Projections démographiques pour le Bénin, Projet IMPETUS, Atlas, Bénin.

INSAE, RGPH3. 2004. Cahier des Villages et Quartiers de Ville, Département de l'Atlantique. RGPH3: République du Bénin, 35p.

LAKANY. 2004. Forêt et initiative de développement. Acte de l'atelier international sur la foresterie (16 février 2004 Rome/ Accra Ghana) FAO, Rome 99p.

PNUD. 2010. Etudes des Ecosystèmes naturels dans le monde: Rapport annuel, PNUD, 195p + annexe. 